\title{
AOR
}

Selected Papers of \#AolR2021:

The 22nd Annual Conference of the

Association of Internet Researchers

Virtual Event / 13-16 Oct 2021

\section{THE NEW SELFIE STANDARD: FACETUNE AND THE SHIFT TOWARD EAST ASIAN SELFIE AESTHETICS}

\author{
JD Swerzenski \\ University of Massachusetts Amherst \\ Dasol Kim \\ University of Pennsylvania
}

\section{Introduction/ Theoretical Framework}

Since its release in 2013, Facetune has risen to become the top paid photo and video app in the US (Sensor Tower, 2021). In allowing users to "retouch selfies" through their "own personal makeover studio," Facetune may seem in line with other photo enhancement apps like VSCO, Instagram, or Snapchat. Yet as we document, the types of retouching tools Facetune make available mark a more significant shift in the American cultural practice of image editing, signaling an adoption of East Asian attitudes toward beauty standards and the plasticity of the image.

We trace this shift through two key cultural beliefs that have underpinned American photo editing discourse. First is the realist view of photography, in which the image is understood as offering a reflection of the real world (Bazin \& Gray, 1960). Second is the conception of cultural imperialism, in which mass media technologies project American cultural standards out to the rest of the world (Schiller, 1991). While both beliefs are grossly oversimplified (Sontag, 1977; Kraidy, 2002), they have produced real impact in East Asian countries, with women in particular being subjected to unrealistic western beauty standards while also facing discipline for not projecting a 'real' self (Leem, 2016). Using a Critical Technocultural Discourse Analysis (CTDA), we highlight how this friction resulted in differing East Asian cultural beliefs around technological practice (Brock, 2018). Procedures such as plastic surgery that are stigmatized in the U.S. have been accepted as tools of upward mobility in South Korea and increasingly China (Wong, 2018). We find the practice of image editing in East Asia follows a similar logic: as transformations allowing one to achieve a better self.

While American platforms have sought to uphold the realist view, with Instagram even "removing all plastic surgery-style tools" in 2019, popular Chinese and Korean apps like BeautyPlus and SNOW approach the image as a plastic object (Spark, 2019). For many East Asian influencers, editing is a "a matter of ordinary courtesy"; users often spend an 
hour editing a single image (Fan, 2019). Facetune has gained success by mirroring the architecture of popular Chinese and Korean apps and marketing its platform toward non-East Asian users. American users have taken to the app for its plethora of digital reconstructive tools not available through mainstream American apps such as Instagram or SnapChat (Solon, 2018). Use of the app has been something of a dark secret, with celebrities like Kylie Jenner accused of Facetuning their images and trying to pass off the results on Instagram. Yet the open embrace of Facetune by influencers like James Charles marks a possible shift away from needing to uphold the realist standard at all.

\section{Method}

Working within the CTDA framework, we track this shift by undertaking a Walkthrough Analysis of the Facetune app. Walkthrough offers a means of engaging directly with the interface and technical functions of the app to "understand how it guides users and shapes their experiences" (Light et al., 2016, p. 2). We both conducted independent walkthroughs of the free Facetune 2 app using our respective smartphones, using our own selfies to test each function. Our walkthrough observations focused on how Facetune redirects the two American beliefs at the core of this study: challenging cultural domination of Western beauty standards and undermining the realist conception of the image. Working with our own images in the Walkthrough also allowed us to reflect on our positionality as a Korean cis-woman and white American cis-man.

\section{Discussion/ Conclusion}

Our findings highlight a number of technical and rhetorical means by which Facetune orients the user. Among the most prominent technical tools was the use of the slider function, used throughout the app as a means of applying editing effects. Though typically set in a neutral middle position, the slider is often placed to one side of the screen in order to direct the user toward a correct application of the effect. For example, the Iris tool only allows the user to increase the size of their irises, signaling the user toward the desired trait. Like all tools, the Iris feature provides a help function, offering a before-and-after-style gif to model correct application of the effect.

We did find evidence of Facetune pushing certain East Asian beauty ideals that may fall outside of American standards. The Face resizing features-allowing users to adjust nose, eyes, forehead, and lips by size and position-speak to a Chinese and Korean conception of proportionality achieved through plastic surgery style alterations (Abidin, 2016). Further, the naming of tools like heal, bandage, cleanse, and fix connect with an East Asian conception of plastic surgery as a healthful, restorative practice (Wong, 2018).

More convincing is the degree to which Facetune presents a full rift with a realist view of photography. After using the retouch, resizing, reshape, and touch up features on our own selfies in the walkthrough, we quickly found a disconnect formed between our real selves and the version we were editing on Facetune. The surrealism of these features extended beyond enhancing the real. Among the most interesting features in our walkthrough was the smirk tool, which allows users to contort the mouth into a smile. Additional functions can alter the lighting direction of a scene or replace the background all together with a digital backdrop. In each case, Facetune uses the photo not in its 
traditional role as a vessel for depicting a certain scene in time, but as a blank canvas on which to produce an ideal digital self. We believe these findings offer a significant contribution in understanding shifting image editing practices online, particularly by highlighting the new technological tools and cultural beliefs that are leading this shift.

\section{References}

Abidin, C. (2016). "Aren't These Just Young, Rich Women Doing Vain Things Online?": Influencer Selfies as Subversive Frivolity. Social Media + Society, 2(2), 1-17.

Bazin, A., \& Gray, H. (1960). The ontology of the photographic image. Film Quarterly, 13(4), 4-9.

Brock, A. (2018). Critical technocultural discourse analysis. New Media \& Society, 20(3), 1012-1030.

Leem, S. Y. (2016). The dubious enhancement: Making South Korea a plastic surgery nation. East Asian Science, Technology and Society: An International Journal, 10(1), 51-71.

Light, B., Burgess, J., \& Duguay, S. (2018). The walkthrough method: An approach to the study of apps. New media \& society, 20(3), 881-900.

Kraidy, M. M. (2002). Hybridity in cultural globalization. Communication theory, 12(3), 316-339.

Schiller, H. I. (1991). Not yet the post-imperialist era. Critical Studies in Media Communication, 8(1), 13-28.

Sensor Tower (2021, April 13). Top Charts: iPhone- US - Photo \& Video - Apr 13, 2021. https://sensortower.com/ios/rankings/top/iphone/us/photo-video?date=2021-04$\underline{13}$

Solon, O. (2018, March 9). FaceTune is conquering Instagram - but does it take airbrushing too far? The Guardian. https://www.theguardian.com/media/2018/mar/09/facetune-photoshopping-appinstagram-body-image-debate

Sontag, S. (1977). On Photography. Farrar, Straus and Giroux.

Spark AR Creators (2019, October 18). Update on Well-Being Policies [Post]. Facebook. https://www.facebook.com/groups/SparkARcommunity/permalink/733132997098 $\underline{745 /}$

Wong, K. Y. J. (2018). No More Taboo: Discursive tactics for navigating the taboo of cosmetic surgery. Global Media and China, 3(4), 271-296. 